ICAASET-2021, 20-21 May, 2021, K.R. Mangalam University, Gurugram

International Journal of Technical Research \& Science (Special Issue)

ISSN No.:2454-2024 (online)

\title{
COMPARATIVE STUDY OF OPTIMIZED LOAD BALANCING MODELS USING FOG- CLOUD NETWORKS
}

\author{
Asha Sohal, Ramesh Kait \\ E-Mail Id: asha.sohal24@gmail.com, ramesh.kait@gmail.com \\ Kurukshetra University, Kurukshetra, Haryana, India
}

\begin{abstract}
Fog computing helps in storing data near to end users and it is taken as the modified level of cloud computing. There are many cloud platforms where we can store the data but because of some limitations, lots of data could not be processed on time. Apart from that, there are various challenges in terms of load balancing over cloud which we need to handle very carefully sothat there is no congestion, less energy consumption and minimum delay in processing. With the help of optimized load balancing models and algorithms, we can increase the productivity of fog-cloud networks. The main objective of this study is to compare the results of load balancing algorithms which creates warehouse of data at very fast access rate to the mobile users. At present scenario, this can be easily done using fog computing in very accessible way. It is an interface between end users and cloud layer and provides higher data access and retrieval services to the end users. This paper includes architecture of cloud computing and its attributes which is necessary to concentrate on the interplay and coordination in between fog and cloud layer. This paper also focuses on some important parameters which are considered in minimizing load over fog-cloud and try to improve the data access rate.
\end{abstract}

Keywords: Fog-Cloud computing, optimization, delay in power or energy consumption, workload distribution.

\section{INTRODUCTION}

The main motive of Digital India concept is basically in the field of services used in electronic, online products, online manufacturing and online job opportunities. Based on this we have large amount of data on daily basis. For this purpose, there is a need of data storage. Now we find a suitable platform for data storage i.e. Cloud. In general, Cloud Computing is basically a new generation technique where we can share our data over one platform. As per the requirements of the users, the technology is growing fast. This most popular archetype of cloud computing is imploring many vendors which increases its demand. According to National Institute of Science and Technology (NIST) [1] Cloud Computing is a model for enabling convenient, on-demand network access to a shared pool of configurable computing resources. It includes various parameters like data related to network, data based on server, data storage, applications and different cloud services that can be promptly provisioned. Also they released with least managerial efforts or with the help of different cloud service provider involvement. It is concluded in 2016 that approximate sixty seven percent of total data storage in conventional IT area will be executed over cloud by using IaaS, PaaS and SaaS. Therefore, by using surging mobile based data traffic rate generated in past few years, we can say that the transfer of data from one point to another point over cloud cannot be possible. It is huge amount of data which require reliable setup over cloud and still it resulted in intolerable transmission delay and less reliable services to end users [2]-[4]. Apart from this real-time communication and lesser execution time with end users and traffic rate is increasing day by day. The concept of mobility and geo-distribution is also critical [5]-[7]. Therefore, use of cloud is becoming so difficult in case of centralized information data storage, data retrieval and management of data over cloud. Nowadays we can say that huge amount of data is generated on daily basis because of mobile devices. It plays very critical role over cloud. It is a hub of gathering information at one place, the success rate of integration of data over cloud. To overcome the major problems in cloud we are focusing on some important parameters like balancing of workload and consumption of power. Therefore, Cisco has explained the purpose of fog computing in 2014, whose motive is to process related workload in sections and provide services locally on fog devices like hardened reliable routers, advanced switches, IP video cameras, etc. instead of data directly transfer to cloud [8]. In short we can also conclude that fog layer can support and entertain many applications which are somehow difficult to cloud layer. There are various applications on which we have to fix up our mind sothat we can provide better productivity like applications which require less and known latency time such as online gaming and video conferencing, applications based on distribution over geographically such as monitoring in pipeline and mobile sensor networks, High access rate mobile based applications like smart connected vehicles and distribution over large scale systems like smart power distribution and smart concept of traffic lights [9]-[12].

In this paper, we consider a fog-cloud computing networking system. Also we are adding some important features like huge amount of data and al time increase in service requests given by users, the energy utilization on powering up and cooling down the cloud servers are soaring these days. It is equally important and necessary to maintain balance in energy management over the fog-cloud computing system [19] [20]. It is also important to provide and guarantee to end users for maintain quality of service throughout the transmission of data. Now we can focus on 
ICAASET-2021, 20-21 May, 2021, K.R. Mangalam University, Gurugram

International Journal of Technical Research \& Science (Special Issue)

ISSN No.:2454-2024 (online)

major parameters one by one and the main point of concern over the fundamental balancing in between the energy utilization and certain delay in the fog-cloud networking system. In this paper, firstly, we study the energy utilization mathematical function and time delay function in case of three subsystems as divided in fig 1 . Then create the concept and working of data assignment over each layer. We can assume that this pioneering work can thoroughly focus on how the fog layer manages the task and complement services to the cloud. The summarized form of this paper has three steps: (i) design and study of a mathematical structure to analyze the energy utilization and time delay issues over fog and cloud layer individually. This gives a better platform where we can say that performance of each system is increased by equal distribution of load and rest of the workload can shift over the other processors timely. Here we are defining the workload of specific machine which means the total time taken for execution of all tasks based on the load assigned to the particular machine [13]-[15]. For implementation of this work we need some mathematical functions based on linear programming, resources used in cloud setup on the basis of load balancing. Load balancing plays an important role over fog-cloud networking system. In this we have a load balancer which generally accepts random multiple requests by the clients and after that it distributes all the requests on multiple servers, networking devices or computers. This also helps in preventing a heavy server or networking device from getting overwhelmed with multiple requests and to assigning the task. There are many examples where we can say that in one system user can send important application request to cloud server and at that moment the cloud server is busy in handling another requests of users, then the process goes into waiting state and server sits idle for that particular time[16]-[18]. Here the users have to wait until the process is fully executed. Now the question arises how we can sort out this problem. For this, we have to keep an eye on the full utilization of server and try to execute the user request first (ii) Try to develop a layout which further divide the prime issue into further subsystem and start working over them individually (iii) Apply testing rules over fog to show fog can perform so well and give complements to the cloud. It also reduces the transmission latency during the execution.

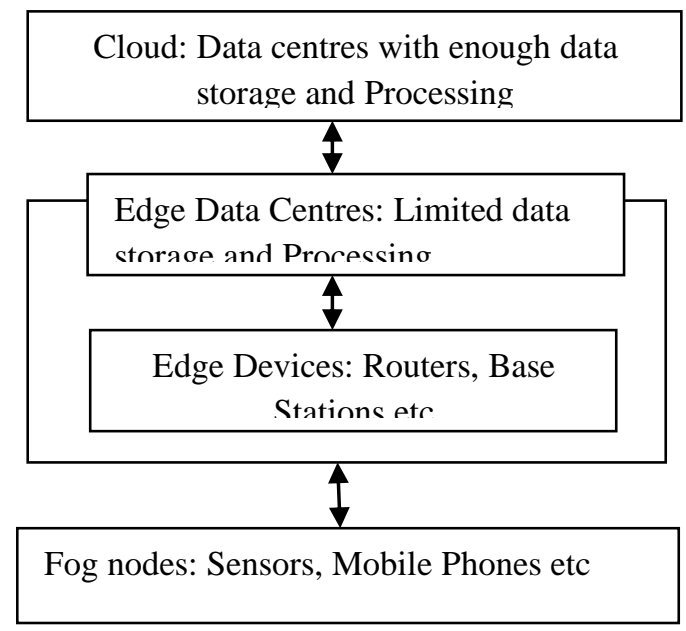

Fig. 1.1 Three Layer Architecture of Fog-Cloud Networks

In fig. 1.1, we showed three-layer architecture of fog-cloud networks which includes cloud layer, edge data centres, edge devices and fog nodes layer. We considered data between cores to edge. Generally, we can say that Fog is "cloud closer to ground" and we can easily share and fetch our data over cloud time to time without any hurdles. There are many ways to do the same in efficient way. With the help of fog computing, things can be accessed easily very closer to the network like latency based sensitive data and some data like delay-tolerant and computational insensitive data can be processed over the cloud. However, we can say that fog layer helps in reducing the load and power consumption over the cloud. The most important things over cloud are computing, data storage, and networking services and the fog extends all the facilities near to edge which enhances the performance of all IoTs [4]. Moreover, the fog based layer provides extra valuable advantages, such as less latency rate, execution and availability of things are closer to the edge of users which is known as fog nodes. They also help in processing of data at specific locations rather than on data centres. It follows the concept of virtualization and distributed system sothat there should be maintain equilibrium at the end of users.

\section{LITERATURE SURVEY}

Morteza Maleki et al (2002) discussed how to increase the lifespan of related networks which is defined by the fixed condition that a fixed amount of percentage of the networking nodes in the network, called die out because of lack of power, transactions related to networks by which each and every mobile node should be controlled so that the energy radiates the same rates all over the nodes [21]. They considered that all networking nodes start with a finite amount of battery capacity and that the energy dissipation per bit of data and control packet transmission or reception is known, they presented an enhanced source-initiated (on demand) routing protocol for MANETs that increases the lifespan of network. Simulation output showed that the proposed "Power-aware Source Routing" protocol can increase the lifespan of network up to thirty percent. Next they approached a greedy policy which was 
ICAASET-2021, 20-21 May, 2021, K.R. Mangalam University, Gurugram

International Journal of Technical Research \& Science (Special Issue)

ISSN No.:2454-2024 (online)

used to fetched links from cache to make sure that no link was overused and also make sure that each and every selected link has minimum battery cost among all possible path between two nodes.

Lei Rao et al (2012) described the issue related to minimization of sum of electricity rate under various electricity environment based on market basis. They discussed about quality of service to the diversity of location and diversity in time of electricity value. The issue was handled by designing a popular model based on mixed integer programming and also proposed an efficient solution algorithm. They found a valuable and related problem of the sum of electricity rate for IDC based service providers by considering various electricity based market setup [19]. They used mixed-integer approach to optimize the cost formulation by using linear programming. After that problem was solved with the help of Brenner's fast polynomial-time method sothat they gained the solution.

Xiumin Wang et al (2014) discussed about the shortcoming of minimization and also convert weighted bipartite matching problem in an auxiliary bipartite graph which helped in reducing the bound of time delay for a specific period. For the general case of the problem, we also design an efficient heuristic algorithm. Finally, implementation results explained about the effectiveness of the suggested scheme. They generate a two-objective problem based on optimization, which was truly on the basis of time delay and migration in cost. For a specific case, every VM demanded homogenous size of resources [24]. They proved that the mentioned issue can be converted into finding a minimum weight bipartite matching in an auxiliary bipartite graph. After that, they derived a lower bound of the minimization time delay for a special setting. An efficient and advanced heuristic algorithm was designed for the general case of the shortcoming.

Ivan Stojmenovic et al (2014) described the state-of-the-art based on Fog computing and many work has done under the similar umbrella. They discussed about two major factors which are categorized as Security and privacy under Fog computing paradigm. They took an example and discussed about many networking serious attacks like man-in-the-middle attack which was very harmful in case of security over Fog computing paradigm [25]. They investigated different features of this attack by analyzing its processing speed and data utilization over Fog based devices and many advantages came out after applying this methodology in several domains. Based on the research work of this valuable paper, some important innovations in computing and data storage may be inspired in the future to tackle data intensive services based on the interplay between Fog and Cloud. Future work will definitely expand on the Fog based computing paradigm into important features of smart grid and software development networks.

Faraz Ahmad et al (2010) proposed a technique named as PowerTrade, which was used to balance in between the idle energy and cooling energy for each other. At the end they reduced the total cost of energy. There was one more important parameter that is related to response time, for this they proposed SurgeGuard which means to overprovision the number of active servers beyond that needed by the current loading so as to absorb future increases in the loading. It was based on two-tier architecture scheme which has given us how to provide balanced energy at coarse time granularities around one hour sothat it played fine-grain replenishment and also absorb the workload increased during the task. This provisioned reserves at fine time granularities around five minutes which handle the uncommon and intolerable abrupt loading based surges. In past, we studied many cooling optimizations techniques which successfully reduced the power cost, distribute the workload all over the servers to achieve equal temperature, but this increased the idle energy by keeping all the servers in active mode [26]. Moreover, many optimizations techniques reduced their response times because of standby-to-active movement delays in time.

Ju Ren et al (2015) discussed about the fluctuated rates in energy harvesting and price in switching of channel. On the basis of these parameters they designed a maximization utility based network approach using mix-integer and non-linear problem in programming and they tried to solve it in effective way then decoupled this pattern by means of advanced dual decomposition. They also proposed JASC, a scheme based on joint channel access, control scheme in rate of sampling. There was an important role of real-time sensors based channels and prices in energy harvesting [27]. They have also proposed a joint channel access and sampling rate control scheme, named JASC, for utilizing the real time sensing results to balance the advanced and dynamic channel access and prices in sampling for sensor based nodes. Implementation results proved that the proposed JASC can gain an enhanced network based utility than available methods. For the future perspective, we will study CRSNs with advanced dynamic routing techniques, where the access rate of channel and rate of control in sampling must be adaptive to the dynamically changed the routing links.

Ashkan Yousefpour et al (2017) discussed about basic architecture of many applications on the basis of cloud, fog and Internet of things (IoTs) and their motive of the research is to minimize the delay in services of IoTs so that they can get less load over system and tried to build congestion free framework. For this purpose, they need to build analytical approach to work on polices and how the proposed architecture helps to reduce IoT service delay. They also discussed about other fog computing policies [28]. They tried to find out offload of a task which was independent on concept of queuing and in future they will try to build fog-cloud framework using Markovian queuing system that would be more cost effective in nature.

Milena F. Pinto et al (2019) discussed about newly formed technology UAV (Unmanned aerial vehicles) which was proposed to perform on a cooperative environment to gear up critical decisionmaking situations. The information in between the aircraft and ground stations was limited by long distances. The new state of art architecture has been used to improve latency, balancing in load, scalability and efficiency to meet time constraints using fog- cloud computing. This research work introduced a model to investigate the fog-cloud computing cooperation to overcome the throughput and latency involving multiple aircraft in areas with low communication 
ICAASET-2021, 20-21 May, 2021, K.R. Mangalam University, Gurugram

International Journal of Technical Research \& Science (Special Issue)

ISSN No.:2454-2024 (online)

infrastructure. Besides, the analyzed model includes the load balancing limitations of UAVs in the proposed architecture [29]. This developed architecture assigns a UAV as head coordinator in a fog computing level to process and to control the communications between the nearest nodes and the cloud.

Judy C. Guevara et al (2020) described about framework of Fog-based computational resources and their integration with the Cloud introduces new challenges in resource management, which requires the implementation of new strategies to guarantee compliance with the quality of load balancing algorithms. They also proposed the implementation of a typical machine learning classification methodology to differentiate many Fog Computing applications as a function of their QoS requirements such as load balancing and less power consumption.

\section{MATHEMATICAL MODEL OF THE SYSTEM}

\subsection{Energy Management based on data usage over Cloud Computing}

There are various cloud servers acts as a host which is based on many homogeneous computing machines. Their specific configurations based on CPU frequency are considered as same for all homogenous machines showing at homogenous server. So, each and every machine at homogenous server has same energy utilization concept. On the basis of these factors, we assume the energy utilization factor of each machine over the cloud based server with a mathematical function of machine and also considering various parameters based on frequency of CPU. Now we can check the smooth functioning of the setup. After that we can increase the work allocation and then we require more cloud based servers for execution. On the other hand, when we see that there is less number of work allocation over cloud then automatically we can off the cloud servers, this will help us in energy saving. To implement this problem on MATLAB, we assume some variable 0/1 which means sever is off/on respectively. Also use some integer variable which represents number of used machines over cloud servers. After that we can find out the energy consumption rate by multiplying off/on situation of server, turn on state of machine number and utilization value of each machine used over cloud setup.

\subsection{Energy Management based on data usage over Fog Computing}

Every fog device uses a mathematical function to compute the energy utilization which is monotonic in nature. This can increase the computation amount and try to solve this method by convex function which is the part of linear programming. Apart from this we can also use piece wise approach based on linear function and quadratic approach. Although, the fog computing machines can adjust any type of energy utilization mathematical functions which satisfies mainly two conditions: (i) Whenever we increase the computation rate of energy utilization, it always increase the computation (ii)There is always increase in marginal energy utilization for every fog devices. To implement these factors we use some mathematical functions and try to find out the optimized solution in this context.

\section{HOW TO INCREASE QUALITY OF SERVICE OVER FOG-CLOUD NETWORKS}

\subsection{To minimize time delay with least energy utilization over Fog Computing}

For this approach, there are different setup in which we are trying to balance between energy utilization and time delay in computation which are important features of fog machines. To implement this concept mathematically we form two different subsystems. In first approach, we assume some adjustable weighting parameter sothat we can balance in between energy utilization and time delay for any fog device. This problem can be solved by convex function by assuming workload $\mathrm{X}$ for the fog subsystem with some linear constraints. Also interior-point method helps in finding the optimized solution corresponding to workload assigning to fog devices in distributed manner. By considering mentioned mathematical function we can calculate the energy utilization and time delay over the fog devices in different subsystem. By this methodology we can maintain the quality of service over fog devices.

\subsection{To minimize time delay issues with least energy utilization over cloud computing}

In this case, we strictly follow service level agreement (SLA) for each and every request received over cloud. There response time must follow specific threshold value sothat there is no confusion in between handling requests over cloud. Else there will be loss in business revenue. We consider that response time delay must be lesser than an adjustable weighting parameter which could be treated as the threshold point in time delay which basically judges the revenue/penalty area.

Table-4.1 Comparison of Different Optimization Load Balancing Models based on Fog-Cloud Networks

\begin{tabular}{|c|l|l|l|c|}
\hline $\begin{array}{l}\text { Authors } \\
\text { Year }\end{array}$ & Proposed Model & $\begin{array}{l}\text { Simulation } \\
\text { Tools Used }\end{array}$ & Performance Metrics & Conclusion \\
\hline $\begin{array}{c}\text { Yichuan Jiang, } \\
2015\end{array}$ & $\begin{array}{l}\text { On the basis of task allocation } \\
\text { and algorithms used in load } \\
\text { balancing, they proposed many } \\
\text { control models, various resource } \\
\text { optimization techniques, } \\
\text { reliability, synchronization and } \\
\text { considered different networks } \\
\text { parameters. }\end{array}$ & Survey report & $\begin{array}{l}\text { There were various } \\
\text { parameters of analyzing } \\
\text { performance like pool of } \\
\text { resources, low reliability } \\
\text { of systems, heterogeneous } \\
\text { nodes, } \\
\text { Different domain of } \\
\text { networks parameters }\end{array}$ & - \\
\hline
\end{tabular}


ICAASET-2021, 20-21 May, 2021, K.R. Mangalam University, Gurugram

International Journal of Technical Research \& Science (Special Issue)

ISSN No.:2454-2024 (online)

\begin{tabular}{|c|c|c|c|c|}
\hline $\begin{array}{l}\text { MortezaMaleki, } \\
\text { KarthikDantu, } \\
\text { and } \\
\text { MassoudPedram } \\
\text {, } 2002\end{array}$ & $\begin{array}{l}\text { There was a comparison of } \\
\text { algorithms between PSR } \\
\text { (Power-aware Source Routing) } \\
\text { algorithm and DSR (Dynamic } \\
\text { Source Routing). Also used } \\
\text { another popular Ad Hoc routing } \\
\text { technique. }\end{array}$ & ns-2 & Network lifetime & $\begin{array}{l}\text { With the help } \\
\text { of PSR } \\
\text { protocol, it } \\
\text { increased the } \\
\text { network } \\
\text { Lifetime up } \\
\text { to } 30 \% \text { using } \\
\text { greedy } \\
\text { policy. This } \\
\text { showed in } \\
\text { simulation } \\
\text { results. }\end{array}$ \\
\hline $\begin{array}{l}\text { Lei } \text { Rao, } \\
\text { Liu, } \\
\text { Wenyu } \\
2012\end{array}$ & $\begin{array}{l}\text { They modeled constrained } \\
\text { mixed-integer programming and } \\
\text { many enhanced algorithm to } \\
\text { minimize the sum of power } \\
\text { utilization cost by considering } \\
\text { various electricity based } \\
\text { markets platform and also } \\
\text { provided QoS to the locale and } \\
\text { time diversity of electricity } \\
\text { price. }\end{array}$ & $\begin{array}{l}\text { Brenner's fast } \\
\text { polynomial- } \\
\text { time } \\
\text { algorithm }\end{array}$ & $\begin{array}{l}\text { With the help of energy } \\
\text { management in IDCs, they } \\
\text { minimized cost flow }\end{array}$ & $\begin{array}{l}\text { Cost reduced } \\
\text { in various } \\
\text { electricity } \\
\text { based market } \\
\text { environment } \\
\text { for IDCs }\end{array}$ \\
\hline $\begin{array}{l}\text { Xiumin Wang, } \\
\text { Xiaoming Chen } \\
\text { Yuen, Weiwei } \\
\text { Wu2014 }\end{array}$ & $\begin{array}{l}\text { They formulate two different } \\
\text { objective optimization } \\
\text { techniques which helped in } \\
\text { balancing the resolution } \\
\text { between time delay and transfer } \\
\text { cost. By using heuristic } \\
\text { algorithm minimization of time } \\
\text { delay and transfer cost based on } \\
\text { specific weight parameter. }\end{array}$ & $\begin{array}{l}\mathrm{C}++, \text { ILP } \\
\text { CPLEX in } \\
\text { NEOS server }\end{array}$ & $\begin{array}{l}\text { They minimized delay and } \\
\text { price of virtual machines } \\
\text { when homogenous size of } \\
\text { resources on weighted } \\
\text { bipartite graph. }\end{array}$ & - \\
\hline $\begin{array}{lr}\text { Ivan } & \text { Stojmeno, } \\
\text { Sheng } & \text { Wen } \\
2014 & \end{array}$ & $\begin{array}{l}\text { Considered different denial } \\
\text { networking attack to provide } \\
\text { security in } \text { Fog-cloud } \\
\text { networking setup. Also } \\
\text { investigated various important } \\
\text { points of these attacks by } \\
\text { analyzing its processing speed } \\
\text { and } \\
\begin{array}{l}\text { Storage utilization over Fog } \\
\text { based tools. }\end{array}\end{array}$ & $\begin{array}{l}\text { Survey } \\
\text { Report }\end{array}$ & $\begin{array}{l}\text { They used some } \\
\text { inventions in computing } \\
\text { and data storage for the } \\
\text { better use of Fog and } \\
\text { Cloud Layer. }\end{array}$ & - \\
\hline $\begin{array}{l}\text { Faraz } \text { Ahmad } \\
\text { and T. N. } \\
\text { Vijaykumar, } \\
2010\end{array}$ & $\begin{array}{l}\text { They proposed } \\
\text { PowerTrade, which resolved } \\
\text { idle power/energy and cooling } \\
\text { power/energy for each layer. } \\
\text { Also used SurgeGuard to } \\
\text { resolve the issue of response } \\
\text { time. }\end{array}$ & $\begin{array}{l}\text { AirPAK, } \\
\text { CFD } \\
\text { computational } \\
\text { fluid } \\
\text { dynamics }\end{array}$ & $\begin{array}{l}\text { Used SurgeGuard, to } \\
\text { alleviate response time } \\
\text { degradation, PowerTrade } \\
\text { is basically used for a } \\
\text { cooling power and also } \\
\text { find optimized solution to } \\
\text { reduce total cost of the } \\
\text { cooling power by } \\
\text { considering two-tier over- } \\
\text { provisioning scheme }\end{array}$ & $\begin{array}{l}\text { They } \\
\text { described two } \\
\text { tier } \\
\text { phenomenon } \\
\text { based on very } \\
\text { popular over- } \\
\text { provisioning } \\
\text { at its peak } \\
\text { level, } \\
\text { smoothly } \\
\text { Increased } \\
\text { loading and } \\
\text { Provisioned } \\
\text { fine time } \\
\text { granularities. }\end{array}$ \\
\hline $\begin{array}{l}\text { Ju Ren, Yaoxue } \\
\text { Zhang, Ruilong } \\
\text { Deng, } \quad \text { Ning } \\
\text { Zhang, } 2015\end{array}$ & $\begin{array}{l}\text { They modeled JASC which } \\
\text { include joint channel access, } \\
\text { rate control methodology based } \\
\text { on sampling. Also proposed } \\
\text { concept of real time based }\end{array}$ & OMNET++ & $\begin{array}{l}\text { They considered various } \\
\text { energy efficient parameter } \\
\text { of clustering, strategies } \\
\text { In dynamic spectrum, } \\
\text { spectrum utilization for }\end{array}$ & $\begin{array}{l}\text { They } \\
\text { Demonstrate } \\
\text { d JASC could } \\
\text { gain an } \\
\text { modified }\end{array}$ \\
\hline
\end{tabular}


ICAASET-2021, 20-21 May, 2021, K.R. Mangalam University, Gurugram International Journal of Technical Research \& Science (Special Issue)

\begin{tabular}{|l|l|l|l|l|}
\hline & $\begin{array}{l}\text { channel sensors which results } \\
\text { different energy parameter rates. }\end{array}$ & & CRSNs. & $\begin{array}{l}\text { networking } \\
\text { scheme rather } \\
\text { than various } \\
\text { available } \\
\text { techniques. }\end{array}$ \\
\hline
\end{tabular}

\section{CONCLUSION}

On the basis of this survey paper, the perception based on fog-cloud networks, an enhanced paradigm which extends its services very closer to the end users was discussed. Concretely, we studied an algebraic framework for energy utilization delay compensation problem in the fog-cloud networking setup. We described the workload assigning problem and various sub problems on the basis of linear programming, convex function, queuing theory (M/M/1) and different algorithms which increased the QoS in fog-cloud systems. Also we studied how to minimize the delay for dispatch in cloud layer. After that we added all the factors together and finally focused on how the data entered into fog-cloud layer and get communicated in balanced manner. We concluded that this mathematical survey task gives valuable suggestions on the user friendly interaction and cooperation in between the fog-cloud network setup. The survey based work has implemented in a centralized way.

\section{FUTURE SCOPE}

For future perspective, there will be many ways by which we will try to find out optimized results on the basis of distributed architecture. In such cases, the related data or exchange of information will be based on fog-cloud layer and transmission overhead needs to be attentively considered.

\section{REFERENCES}

[1] [1] R. Deng, R. Lu, C. Lai, and T. H. Luan, "Towards power consumption delay tradeoff by workload allocation in cloud-fog computing," in Proc. IEEE ICC, 2015, pp. 3909-3914.

[2] [2] R. Lu, H. Zhu, X. Liu, J. K. Liu, and J. Shao, “Toward efficient and privacy-preserving computing in big data era," IEEE Network, vol. 28, no. 4, pp. 46-50, 2014.

[3] [3] N. Kumar, S. Misra, J. Rodrigues, and M. Obaidat, "Coalition games for spatio-temporal big data in Internet of vehicles environment: a comparative analysis,” IEEE Internet of Things Journal, vol. 2, no. 4, pp. 310-320, 2015.

[4] [4] C. Lai, R. Lu, D. Zheng, H. Li, and X. Shen, "Toward secure large-scale machine-to-machine communications in 3GPP networks: challenges and solutions," IEEE Communications Magazine, vol. 53, no. 12, pp. 12-19, 2015.

[5] [5] T. H. Luan, L. X. Cai, J. Chen, X. Shen, and F. Bai, "Engineering a distributed infrastructure for large-scale cost-effective content dissemination over urban vehicular networks," IEEE Transactions on Vehicular Technology, vol. 63, no. 3, pp. 1419-1435, 2014.

[6] [6] S. He, J. Chen, X. Li, X. S. Shen, and Y. Sun, "Mobility and intruder prior information improving the barrier coverage of sparse sensor networks," IEEE Transactions on Mobile Computing, vol. 13, no. 6, pp. 1268-1282, 2014.

[7] [7] N. Lu, N. Cheng, N. Zhang, X. Shen, and J. W. Mark, "Connected vehicles: Solutions and challenges," IEEE Internet of Things Journal, vol. 1, no. 4, pp. 289-299, 2014.

[8] [8] The Network. Cisco Delivers Vision of Fog Computing to Accelerate Value from Billions of Connected Devices. [Online]. Available: http://newsroom.cisco.com/press-release-content?articleId=1334100

[9] [9] S. He, J. Chen, F. Jiang, D. K. Yau, G. Xing, and Y. Sun, "Energy provisioning in wireless rechargeable sensor networks," IEEE Transactions on Mobile Computing, vol. 12, no. 10, pp. 1931-1942, 2013.

[10] [10] F. Bonomi, R. Milito, P. Natarajan, and J. Zhu, "Fog computing: A platform for Internet of Things and analytics," in Big Data and Internet of Things: A Roadmap for Smart Environments. Springer, 2014, pp. 169186.

[11] [11] R. Deng, Z. Yang, M.-Y. Chow, and J. Chen, "A survey on demand response in smart grids: Mathematical models and approaches," IEEE Transactions on Industrial Informatics, vol. 11, no. 3, pp. 570-582, 2015.

[12] [12] J. Chen, Q. Yu, B. Chai, Y. Sun, Y. Fan, and X. Shen, "Dynamic channel assignment for wireless sensor networks: A regret matching based approach," IEEE Transactions on Parallel and Distributed Systems, vol. 26, no. 1, pp. 95-106, 2015.

[13] [13] L. Atzori, A. Iera, and G. Morabito, “The Internet of Things: A survey,”Computer networks, vol. 54, no. 15 , pp. 2787-2805, 2010.

[14] [14] J. A. Stankovic, "Research directions for the Internet of Things," Internet of Things Journal, IEEE, vol. 1, no. 1, pp. 3-9, 2014.

[15] [15] A. Zanella, N. Bui, A. Castellani, L. Vangelista, and M. Zorzi, "Internet of Things for smart cities," IEEE Internet of Things Journal, vol. 1, no. 1, pp. 22-32, 2014.

[16] [16] S. K. Datta, C. Bonnet, and J. Haerri, "Fog computing architecture to enable consumer centric Internet of Things services," in Proc. IEEE International Symposium on Consumer Electronics (ISCE), 2015, pp. 1-2. 
ICAASET-2021, 20-21 May, 2021, K.R. Mangalam University, Gurugram

[17] [17] T. H. Luan, L. Gao, Z. Li, Y. Xiang, and L. Sun, "Fog computing: Focusing on mobile users at the edge," arXiv preprint arXiv:1502.01815, 2015.

[18] [18] R. Suryawansh and G. Mandlik, "Focusing on mobile users at edge and Internet of Things using fog computing," International Journal of Scientific Engineering and Technology Research, vol. 4, no. 17, pp. 3225-3231, 2015.

[19] [19] L. Rao, X. Liu, L. Xie, and W. Liu, "Coordinated energy cost management of distributed Internet data centers in smart grid," IEEE Transactions on Smart Grid, vol. 3, no. 1, pp. 50-58, 2012.

[20] [20] L. Yu, T. Jiang, Y. Cao, and Q. Qi, "Carbon-aware energy cost minimization for distributed Internet data centers in smart microgrids," IEEE Internet of Things Journal, vol. 1, no. 3, pp. 255-264, 2014.

[21] [21] M. Maleki, K. Dantu, and M. Pedram, "Power-aware source routing protocol for mobile ad hoc networks," in Proc. ACM International Symposium on Low Power Electronics and Design, 2002, pp. 72-75.

[22] [22] L. Rao, X. Liu, M. D. Ilic, and J. Liu, "Distributed coordination of Internet data centers under multiregional electricity markets," Proceedings of the IEEE, vol. 100, no. 1, pp. 269-282, 2012.

[23] [23] S. Yi, C. Li, and Q. Li, “A survey of fog computing: Concepts, applications and issues," in Proc. ACM Workshop on Mobile Big Data (Mobidata), 2015, pp. 37-42.

[24] [24] X. Wang, X. Chen, C. Yuen, W. Wu, and W. Wang, "To migrate or to wait: Delay-cost tradeoff for cloud data centers," in Proc. IEEE Globe com, 2014, pp. 2314-2319.

[25] [25] I. Stojmenovic and S. Wen, "The fog computing paradigm: Scenarios and security issues," in Proc. Federated Conference on Computer Science and Information Systems (FedCSIS), 2014, pp. 1-8.

[26] [26] F. Ahmad and T. Vijay kumar, "Joint optimization of idle and cooling power in data centers while maintaining response time," in ACM Sigplan Notices, vol. 45, no. 3, 2010, pp. 243-256.

[27] [27] J. Ren, Y. Zhang, R. Deng, N. Zhang, D. Zhang, and X. Shen, "Joint channel access and sampling rate control in energy harvesting cognitive radio sensor networks," IEEE Transactions on Emerging Topics in

[28] Computing, DOI: 10.1109/TETC.2016.2555806, to appear.

[29] [28] Ashkan Yousefpour, Genya Ishigaki, and Jason P. Jue "Fog Computing: Towards Minimizing Delay in the Internet of Things" IEEE 1st International Conference on Edge Computing, 2017, DOI 10.1109/IEEE.EDGE.2017.12

[30] [29] Milena F. Pinto , André L. M. Marcato , Aurélio G. Melo , Leonardo M. Honório , and Cristina Urdiales "A Framework for Analyzing Fog-Cloud Computing Cooperation Applied to Information Processing of UAVs" Wireless Communications and Mobile Computing" Volume 2019, Article ID 7497924, 14 pages https://doi.org/10.1155/2019/7497924

[31] [30] Judy C. Guevara, Ricardo da S. Torres, Nelson L.S. da Fonseca, "On the classification of fog computing applications: A machine learning perspective” DOI https://doi.org/10.1016/j.jnca.2020.102596, March 2020.

DOI Number: https://doi.org/10.30780/specialissue-ICAASET021/003 